\title{
Clinical presentation and treatment outcomes of spinal epidural arteriovenous fistulas
}

\author{
Deena M. Nasr, DO, ${ }^{1}$ Waleed Brinjikji, MD, ${ }^{2}$ Michelle J. Clarke, MD, ${ }^{3}$ and Giuseppe Lanzino, MD ${ }^{3}$ \\ Departments of ${ }^{1}$ Neurology, ${ }^{2}$ Radiology, and ${ }^{3}$ Neurosurgery, Mayo Clinic, Rochester, Minnesota
}

\begin{abstract}
OBJECTIVE Spinal epidural arteriovenous fistulas (SEDAVFs) constitute a rare but treatable cause of vascular myelopathy and are a different subtype from the more common Type I spinal dural AVFs. The purpose of this study was to review a consecutive series of SEDAVFs from a single institution and report on the clinical presentations, functional status, and treatment outcomes.
\end{abstract}

METHODS The authors identified all SEDAVFs treated at their institution from 2005 to 2015. SEDAVFs were defined as spinal AVFs in which the fistulous connection occurred in the epidural venous plexus. The clinical presentation, functional status, immediate treatment outcomes, and long-term neurological outcomes were analyzed.

RESULTS Twenty-four patients with SEDAVFs were included in this study. The patients' mean age at presentation was 66.9 years. The most common presenting symptoms were pain and numbness ( 22 patients, $91.7 \%$ ), followed by lowerextremity weakness (21 patients, 87.5\%). The mean duration of symptoms prior to diagnosis was 11.8 months. Eighteen patients $(75.0 \%)$ were treated with endovascular therapy alone, $4(16.7)$ with surgery, and $2(8.3 \%)$ with a combination of techniques. There was 1 major treatment-related complication (4.2\%). Fifteen patients (62.5\%) had improvement in disability, and 12 patients (54.5\%) had improvement in sensory symptoms.

CONCLUSIONS SEDAVFs often present with lower-extremity motor dysfunction and sensory symptoms. With the availability of newer liquid embolic agents, these lesions can be effectively treated with endovascular techniques. Surgery is also effective at treating these lesions, especially in situations where endovascular embolization fails or is not safe and in patients presenting with mass effect from compressive varices.

https://thejns.org/doi/abs/10.3171/2016.9.SPINE16618

KEY WORDS spinal vascular malformation; embolization; vascular disorders

$\mathrm{W}$ ITH improvement in our understanding of the pathological anatomy of spinal vascular malformations and improved imaging techniques, a different entity, spinal epidural arteriovenous fistula (SEDAVF), is diagnosed with increasing frequency. SEDAVFs are the result of a fistulous connection between branches of the paraspinal or paravertebral arterial system and the epidural venous plexus ${ }^{7,8,16}$ and may or may not have secondary, retrograde intradural venous drainage. Patients may therefore develop a compressive myelopathy secondary to venous engorgement or a congestive myelopathy from intradural venous drainage. ${ }^{7,16}$ Like spinal dural AVFs (SDAVFs), SEDAVFs often initially present with vague symptoms, such as leg dysesthesias and exertional leg weakness, that slowly progresses to severe myelopathy with paraplegia and sphincter dysfunction. ${ }^{3}$ However, differentiation between SEDAVFs and the more common and well-established Type I SDAVFs is very important, as therapeutic choices may differ; some authors contend that SDAVFs are better treated surgically while SEDAVFs are better treated with endovascular techniques. ${ }^{20}$

Since SEDAVFs are rare, few studies have sought to systematically investigate the clinical presentation, imaging characteristics, and treatment outcomes. In fact, studies of these lesions are limited to very small case series or case reports. We reviewed a consecutive series of SE-

ABBREVIATIONS AVF = arteriovenous fistula; $\mathrm{mRS}=$ modified Rankin Scale; SDAVF = spinal dural AVF; SEDAVF = spinal epidural AVF.

SUBMITTED May 27, 2016. ACCEPTED September 13, 2016.

INCLUDE WHEN CITING Published online February 10, 2017; DOI: 10.3171/2016.9.SPINE16618. 
DAVFs diagnosed and treated at our institution and report on clinical presentation, functional status, and treatment outcomes.

\section{Methods \\ Patient Population}

Following institutional review board approval, we reviewed a consecutive series of angiographically confirmed SEDAVFs diagnosed and treated at our institution from 2005 to 2015. SEDAVFs were defined as spinal AVFs in which the fistulous connection occurred in the extradural venous plexus as opposed to the nerve root sleeve or intradural/intramedullary compartment. Characterization of fistulas as SEDAVFs was determined by 2 reviewers. Some of the patients included in this series were already described in the past as part of much smaller reports on specific treatment modalities from our institution. ${ }^{6,11}$

\section{Data Collection}

Patient demographic variables collected included age and sex. Every patient had received an extensive evaluation by a neurologist before consideration for treatment. For the purpose of this analysis, patient symptoms at the time of treatment were categorized as pain/numbness, lower-extremity weakness, urinary dysfunction, and bowel dysfunction. Duration of symptoms prior to diagnosis, pretreatment modified Rankin Scale (mRS) and Aminoff motor disability ${ }^{1}$ scores were retrospectively calculated by a vascular neurologist through retrospective review of neurology and physiatry notes. These standardized notes included data on motor function, sensory function, urinary function, pain, ambulation, quality of life, and functional status (i.e., ability to carry out usual duties and activities, ability to look after own affairs without assistance, ability to walk without assistance, ability to attend to own bodily needs without assistance, requirement for nursing care, etc.). We also reported any prior spine surgery and the initial diagnosis prior to the discovery of an SEDAVF.

\section{Treatment Types}

Treatment was categorized as endovascular only, surgical only, or staged endovascular followed by surgical. Endovascular treatment of these fistulas was performed by a multidisciplinary team including 2 neurointerventional radiologists and an endovascular neurosurgeon, all with at least 15 years of experience. Treatment decisions were made by consensus between the neurointerventional radiologists and the endovascular neurosurgeon. If, based on the pretreatment spinal angiogram, the team of neurointerventionalists felt that catheter placement within the epidural venous pouch or just proximal to the pouch was feasible, then the patient went on to receive endovascular treatment. If not, the patient would be triaged to surgery. Surgical treatment was performed by either of 2 experienced neurosurgeons. For endovascular treatment, information was collected on the type of embolic agent used (i.e., Onyx, glue, or particles). Treatment-related complications were categorized as minor if they did not result in additional disability or change management of the patient and major if they resulted in additional disability.

\section{Imaging Evaluation}

All imaging was evaluated by a radiologist with 5 years of experience and a neurosurgeon with 15 years of experience. Imaging characteristics studied included the level of the fistula (i.e., thoracic, lumbar, or sacral), number of arterial feeders, presence of intradural venous drainage and venous engorgement resulting in cord compression, $\mathrm{T} 2$ cord signal, vascular flow voids, presence of a venous pouch at the site of the fistula, and spinal cord enhancement. Posttreatment imaging including spinal MR angiograms and digital subtraction angiograms were evaluated for the presence of complete occlusion of the fistula. Complete occlusion of the fistula was defined as no contrast opacification of the venous extradural pouch or of any associated intradural drainage.

\section{Treatment Outcomes}

Posttreatment outcomes were assessed by a vascular neurologist through retrospective review of neurology, neurosurgery, and physiatry notes. Data on improvement in disability, motor function, bowel symptoms, bladder symptoms, and sensory symptoms were collected. Improvement in motor function was defined as an improvement of at least 1 point in the Aminoff motor disability score. Improvement in disability was defined as improvement of at least 1 point in the mRS. Improvement in bladder, bowel, and sensory symptoms was noted if the patient reported subjective improvement in the clinical notes. The mean duration of clinical follow-up was calculated.

\section{Statistical Analysis}

No statistical comparisons were performed. Continuous variables are reported as mean values with standard deviations and categorical variables are reported as counts and frequencies. All analyses were performed using JMP10.0 (www.jmp.com).

\section{Results}

\section{Baseline Characteristics}

The baseline patient characteristics are reported in Table 1. A total of 24 patients were included in this study (mean age $66.9 \pm 11.3$ years). Fifteen $(62.5 \%)$ of the patients were male. The baseline presenting symptoms included pain/ numbness in 22 patients (91.7\%), lower-extremity weakness in 21 patients $(87.5 \%)$, urinary dysfunction in 17 patients (70.8\%), and bowel dysfunction in 15 patients $(62.5 \%)$.

The mean duration of symptoms prior to definitive diagnosis was $11.8 \pm 13.1$ months. In 7 patients (29.2\%), imaging findings quickly led to the diagnosis of an SEDAVF, and no misdiagnosis was made. In 17 patients (70.8\%), a diagnosis other than SEDAVF was initially made. The most common misdiagnosis was transverse myelitis (in 5 patients [20.8\%]), followed by chronic inflammatory demyelinating polyneuropathy (in 3 patients [12.5\%]). Two patients were diagnosed with spinal stenosis $(8.3 \%)$ and 2 patients were initially diagnosed with spinal neoplasms (8.3\%).

A total of 6 patients underwent spinal surgery prior to the diagnosis of the SEDAVF. In 4 patients, surgery was performed due to misdiagnosis of the cause of the 
TABLE 1. Baseline characteristics

\begin{tabular}{lc}
\hline \multicolumn{1}{c}{ Variable } & Value \\
\hline No. of patients & 24 \\
\hline Mean age, yrs & $66.9 \pm 11.3$ \\
\hline Sex & $15(62.5)$ \\
\hline Male & $9(37.5)$ \\
\hline Female & \\
\hline Presenting Sx & $22(91.7)$ \\
\hline Pain/numbness & $17(70.8)$ \\
\hline Urinary dysfunction & $15(62.5)$ \\
\hline Bowel dysfunction & $21(87.5)$ \\
\hline Lower-extremity weakness & \\
\hline Initial misdiagnosis & $3(12.5)$ \\
\hline CIDP & $1(4.2)$ \\
\hline Discopathy & $5(20.8)$ \\
\hline Myelopathy NOS & $2(8.3)$ \\
\hline Spinal stenosis & $1(4.2)$ \\
\hline Peripheral neuropathy & $1(4.2)$ \\
\hline Neurosarcoidosis & $2(8.3)$ \\
\hline Neoplasm & $1(4.2)$ \\
\hline Multiple sclerosis & $1(4.2)$ \\
\hline Tethered cord & $7(29.2)$ \\
\hline None & $11.8 \pm 13.1$ \\
\hline Mean duration of Sx prior to Dx, mos & $6(25.0)$ \\
\hline Spine surgery prior to Dx & $D x=09 n 5 s$ \\
\hline
\end{tabular}

$\mathrm{CIDP}=$ chronic inflammatory demyelinating polyneuropathy; $\mathrm{Dx}=$ diagnosis; NOS $=$ not otherwise specified; $\mathrm{Sx}=$ symptoms.

Values represent numbers of patients (\%) unless otherwise indicated. Mean values are presented \pm standard deviations.

patient's myelopathy, and in 2 patients surgery was performed prior to development of myelopathic symptoms. Four patients (16.7\%) had underlying neural tube defects, including a spinal lipoma in 1 patient, a tethered cord with spinal lipoma in 2 patients, and partial diastematomyelia in 1 patient.

\section{Imaging Characteristics}

Representative images are provided in Figs. 1-5. The most common location of the SEDAVFs was the lumbar spine (in 15 patients, $62.5 \%$ ), followed by the sacrum (in 7 patients, $29.2 \%)$. Twenty-three patients $(95.8 \%$ ) had intradural venous drainage, and 1 patient $(4.2 \%)$ had compressive symptoms from a large epidural venous pouch. All patients had high T2 signal intensity in the spinal cord. Flow voids were present in $83.3 \%$ of cases (20 patients), and a venous pouch at the site of the fistula on angiography was present in 22 cases (91.7\%).

\section{Treatment Characteristics}

Treatment characteristics and outcomes are summarized in Table 2. In 18 cases $(75.0 \%)$, the lesion was only treated endovascularly. In 4 cases (16.7\%), surgical treatment was performed with the intent to close the fistula. In 2 cases (8.3\%), the patients underwent planned staged treatment with partial endovascular embolization followed by definitive surgical treatment. The overall complete occlusion rate was $91.7 \%(22 / 24)$. The rates of complete or near-complete occlusion were $94.4 \%$ (17/18) with endovascular management alone, $75.0 \%$ (3/4) with primary surgical treatment, and $100 \%(2 / 2)$ with planned multimodality treatment. In 1 case of surgical treatment, the fistula recurred and was retreated with Onyx embolization and completely obliterated. Thus, a total of 21 patients underwent some sort of endovascular treatment. The most commonly used embolic agent was Onyx (19 cases). In the cases of staged endovascular-surgical treatment, particle embolization was performed. Examples of cases in which Onyx embolization was performed are provided in Figs. 1, 2, and 4.

Postoperative complications occurred in 2 cases. In 1 case there was a minor complication of a vertebral body infarct following Onyx embolization. This did not result in any additional disability or change in management. One patient suffered a spinal cord infarction following endovascular embolization with Onyx and experienced worsening of symptoms as a result.

\section{Long-Term Treatment Outcomes}

The mean duration of follow-up after treatment was $12.5 \pm 14.4$ months (range 3-60 months, median 6 months). Overall, 15 patients $(62.5 \%)$ had some improvement in disability and motor function. Five patients (33.3\%) had improvement in bowel function, 12 patients $(54.5 \%)$ had improvement in sensory symptoms, and 7 patients (41.2\%) had improvement in bladder function. The mean $\mathrm{mRS}$ score at treatment was $2.9 \pm 1.2$ (median 3, IQR 2-4). Following treatment, the mean $\mathrm{mRS}$ score of the overall patient population improved to $2.1 \pm 1.3$ (median 2, IQR 1-3) $(\mathrm{p}=0.004)$. The mean Aminoff motor disability score was $3.0 \pm 1.8$ (median 3, IQR 1-5) at the time of treatment and was $2.4 \pm 1.8$ (median 3, IQR 0-4) following treatment. The distribution of mRS and Aminoff scores at the time of treatment and at follow-up is provided in Table 3.

\section{Discussion}

Our study of 24 patients with SEDAVFs highlights a number of important findings regarding the clinical and anatomical characteristics of these lesions. First, these fistulas often present as a result of myelopathic symptoms, including lower-extremity weakness, numbness/pain, and urinary and bowel dysfunction, which are not clinically distinguishable from those of patients with the more common Type I SDAVF. In general, symptoms are present for several months before the diagnosis is made, and as with other forms of vascular myelopathy, patients are often misdiagnosed as having other causes of myelopathy such as spinal stenosis or transverse myelitis. The imaging appearance of SEDAVFs on MRI is similar to that of SDAVFs, with high T2 cord signal intensity, intradural flow voids, and intramedullary enhancement-but with the key differentiating factor of the presence of a venous pouch in the epidural venous plexus. At our institution, a majority of these lesions were successfully treated with endovascular therapy alone with high rates of complete or near-complete occlusion and low rates of complications. 

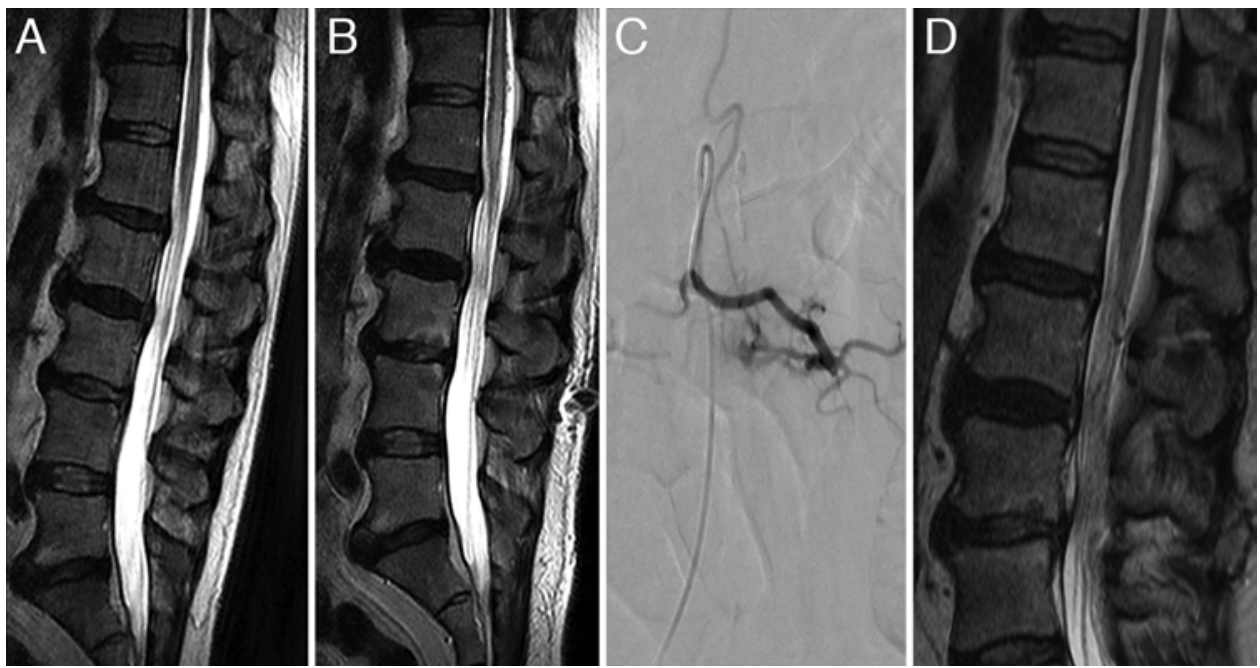

FIG. 1. SEDAVF following discectomy. This 67-year-old man developed sudden-onset left lower-extremity pain and weakness and underwent a discectomy at L-3. The prediscectomy sagittal T2-weighted MR image (A) shows no evidence of intradural flow voids or high T2 cord signal. Six months later the patient reported difficulty standing and walking and became dependent on a cane. This symptomology led to repeat imaging, which demonstrated a new area of high T2 signal intensity in the cord and intradural flow voids (B, sagittal T2-weighted MR image). The patient underwent a spinal angiogram, which demonstrated an SEDAVF (C), which was embolized with Onyx. Postoperative MRI showed a substantial reduction in T2 cord signal intensity and reduction in the extent of intradural flow voids (D, sagittal T2-weighted MR image).
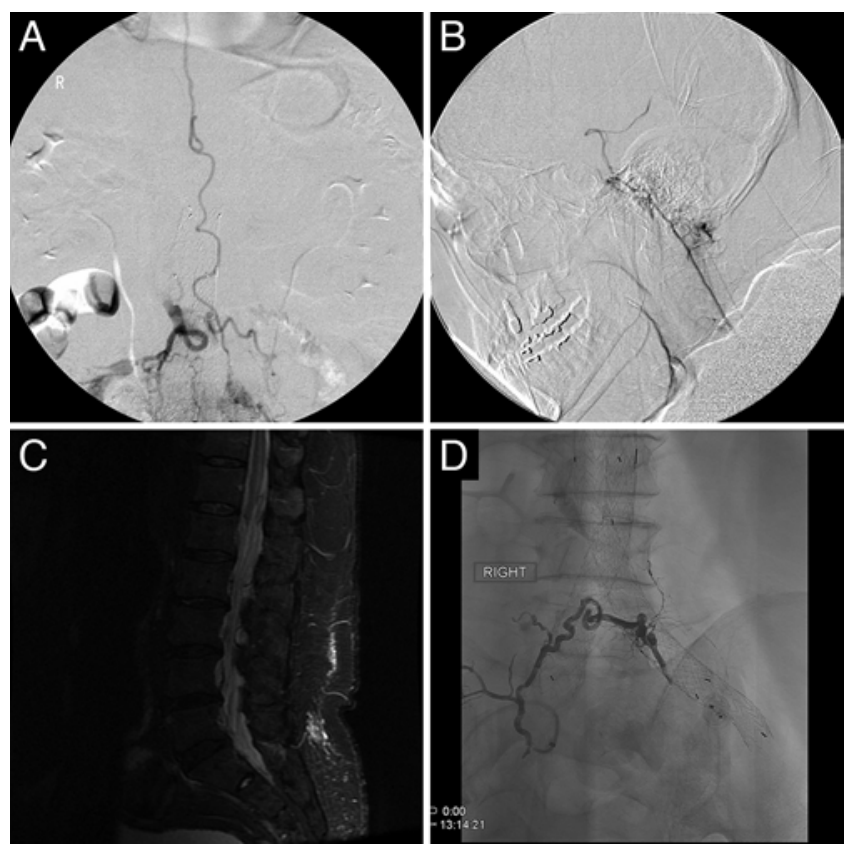

FIG. 2. SEDAVF incidentally detected during angiogram for endoleak following endovascular repair of an abdominal aortic aneurysm. This 74-year-old man had undergone endovascular repair of an abdominal aortic aneurysm and left common iliac aneurysm. Because of growth of the aneurysm on follow-up CT angiography studies, an angiogram was performed to identify a source of an endoleak. Incidentally noted was a large SEDAVF with intradural venous drainage extending to the pontomesencephalic veins and basal veins of Rosenthal (A and B). No treatment was performed at the time as the patient was asymptomatic. Three years later he developed new-onset lower-extremity weakness. Spine MRI showed high T2 signal intensity in the cord with intradural vascular flow voids (C, sagittal T2-weighted MR image). The fistula was embolized with Onyx (D, radiograph) and he experienced improvement in his motor symptoms.
Nearly two-thirds of treated patients experienced some improvement in disability and motor function following treatment, and over half of treated patients had some improvement in sensory symptoms.

\section{Patient Characteristics and Clinical Presentation}

Only a limited number of cases of SEDAVFs have been reported in the literature. As in other forms of spinal fistulas, the clinical manifestations of SEDAVFs are often protean and can progress in a stepwise manner. Because of the variable venous drainage patterns of these lesions (i.e., intradural vs epidural), the clinical manifestations can vary from a myelopathy resulting from intradural venous congestion to a compressive myelopathy resulting from engorgement of the epidural venous plexus. ${ }^{6}$ SEDAVFs can, rarely, present with epidural or intradural hemorrhage, $, 4,-10,12,14,15,17-19,22$ and in some cases the lesions are completely asymptomatic (Fig. 2).

The etiology of these lesions remains unclear. Some have suggested a possible association between spinal surgery and the formation of SEDAVFs. ${ }^{7}$ This is based on the hypothesis that microvascular injuries in the epidural venous plexus during discectomies and other spinal surgeries can cause traumatic AVFs in the epidural space, which can later become symptomatic. In the systematic review by Huang et al., $10 \%$ of SEDAVF patients had surgery prior to diagnosis of the SEDAVF. ${ }^{8}$ In our series, 2 patients (approximately 10\%) had surgery at the level of the fistula prior to the development of symptoms, suggesting that this could have been the etiology in those particular cases (Fig. 1). Four other patients had surgery due to misdiagnosis of the cause of myelopathy, and in those cases the surgery likely did not contribute to the formation of the fistula. As the science currently stands, the vast majority of SEDAVFs remain idiopathic. 

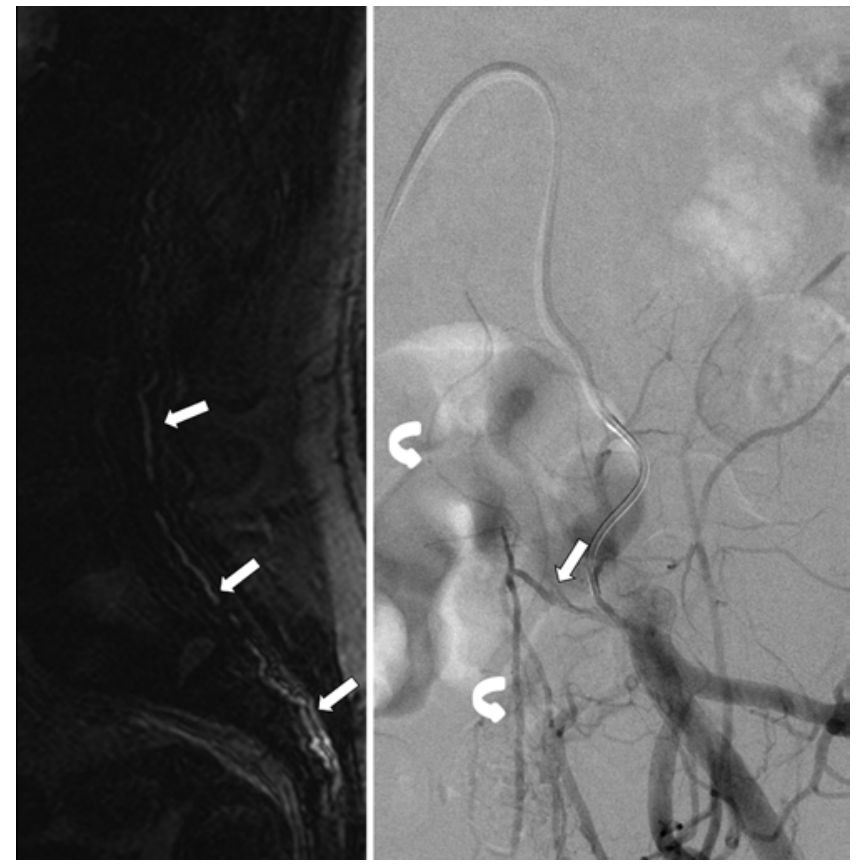

FIG. 3. Sacral epidural arteriovenous fistula. Left: Spinal MR angiogram demonstrating a large dilated intradural vein (arrows). Right: Left iliac angiogram demonstrating a dilated intradural vein coursing up and down the spinal canal (curved arrows). The feeding artery enters at the left S-4 neural foramen (arrow), and there is a small fistulous connection.

SEDAVFs with intradural venous drainage ( $>95 \%$ of the lesions seen in our series) are generally diagnosed in patients around the 6th decade of life. These lesions are seen mainly in the lumbar and sacral regions, ${ }^{6,8}$ unlike Type I SDAVFS, which commonly involve thoracic levels. This distribution is thought to be related to the rich vascular network between the intradural and extradural venous systems in those regions. ${ }^{23}$ Patients with these lesions typically present with myelopathy, sensory and motor radiculopathy, and sphincter malfunction. Transient worsening of symptoms with the Valsalva maneuver (which increases the intradural congestion) is not uncommon in patients with SDAVFS and is more common than in patients with Type I SDAVFs. Because of the risk of irreversible cytotoxic edema in the spinal cord, early diagnosis and treatment are essential to achieve a favorable outcome. ${ }^{13}$ Yet, despite widespread utilization of MRI, the diagnosis continues to be delayed, with a mean time of 11 months from symptom onset to correct diagnosis.

\section{Imaging Characteristics}

Our detailed imaging review of these lesions reveals several interesting features of SEDAVFs that have not received adequate attention in the available literature. First, the imaging characteristics of SEDAVFs on MRI are very similar to those of SDAVFs, with a vast majority of patients presenting with high T2 cord signal intensity, intradural vascular flow voids, and spinal cord enhancement. ${ }^{2}$ However, especially in the sacral fistulas, it is not unusual that the only exuberant intradural flow void noted is a large, arterialized vein ascending from the sacrum to the conus
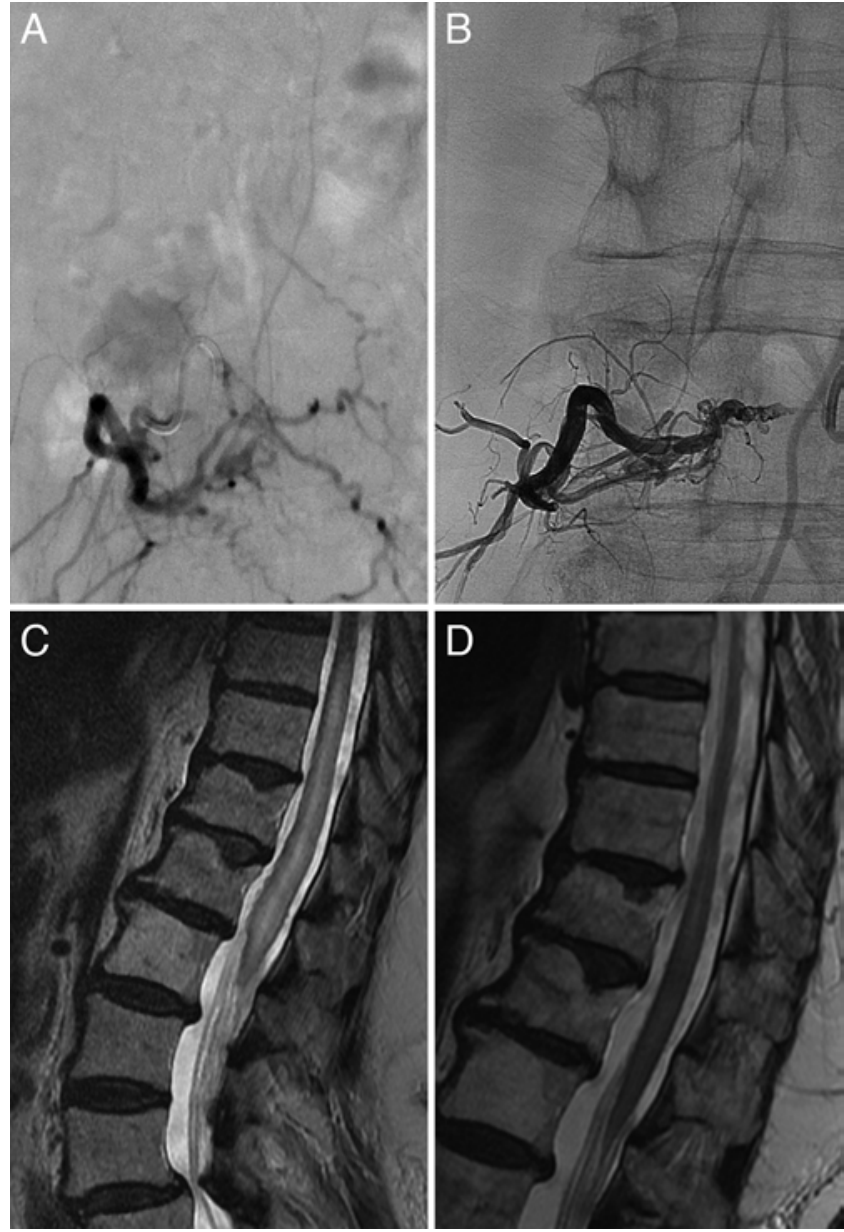

FIG. 4. Lumbar epidural AVF treated with Onyx. This 78-year-old woman presented with bilateral lower-extremity weakness and urinary incontinence. Spinal angiography of the right L-3 radicular artery $(\mathbf{A})$ demonstrated an SEDAVF with a venous pouch in the epidural space and an intradural draining vein. Following Onyx embolization there was complete occlusion of the fistula (B, radiograph). Pretreatment T2-weighted MRI (C, sagittal image) showed high signal intensity and expansion of the conus medullaris. Posttreatment T2-weighted MRI (D, sagittal image obtained 6 months after treatment) showed interval reduction in the T2 signal intensity and amount of cord swelling. The patient reported improvement in lower-extremity strength and improved urinary function after treatment.

(Fig. 3). On spinal angiography these lesions are clearly distinct from SDAVFs and other spinal fistulas in that the location of the fistula is in the epidural venous plexus (Fig. 5), usually associated with a clear venous pouch, which may (in the majority of cases) or may not drain retrograde through an intradural vein. The site of the fistula was well demarcated by an epidural venous pouch in over $90 \%$ of patients in our series. However, in only 1 case was the epidural venous pouch large enough to cause compressive myelopathy secondary to spinal canal narrowing.

\section{Treatment and Outcomes}

Differentiation between SEDAVFs and Type I SDAVFs has very important therapeutic implications. In SEDAVFs, the fistulous connection typically occurs in the ventral epi- 

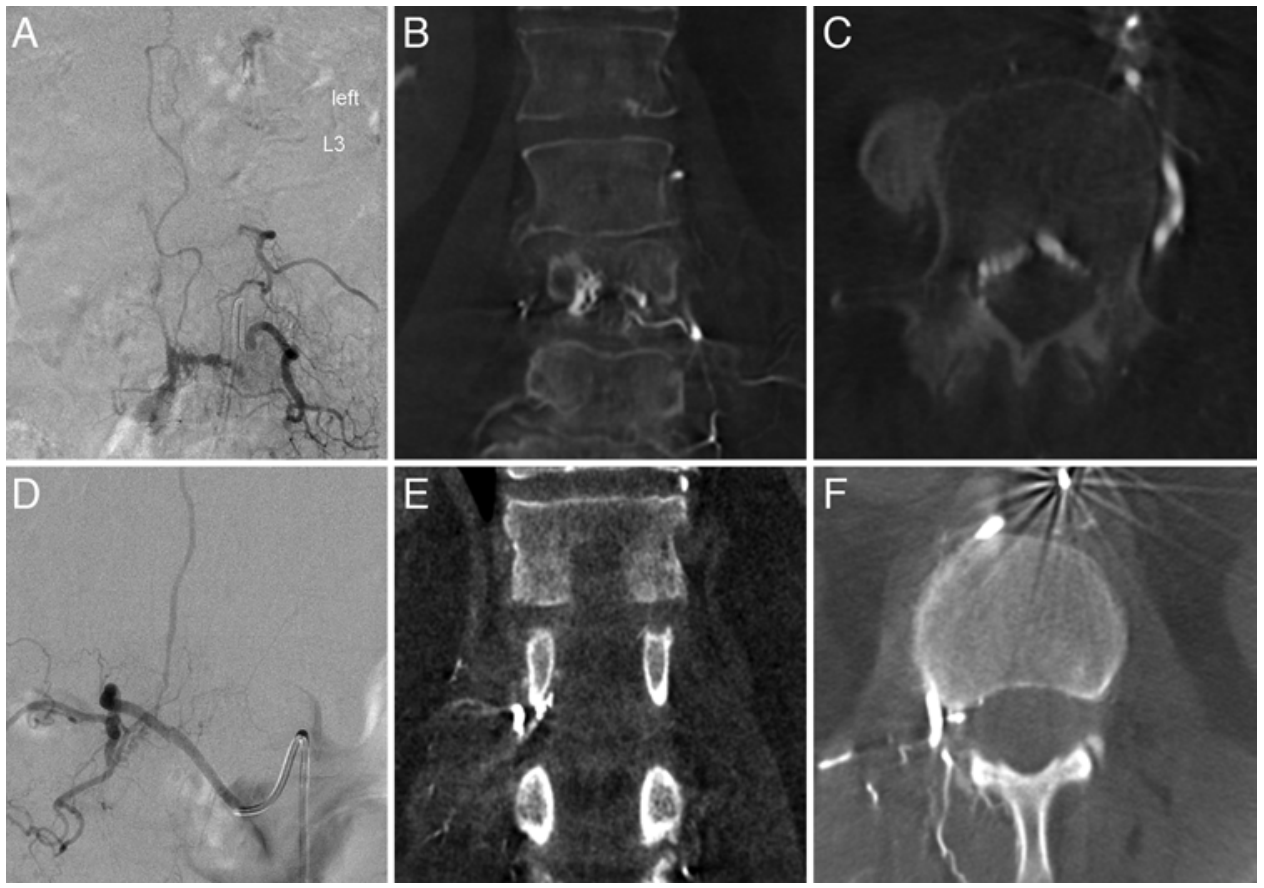

FIG. 5. Difference between SDAVF and SEDAVF. A-C: Spinal angiogram $(A)$ and XperCT images (B and C) demonstrating an SEDAVF fed by the left L-3 lumbar artery. The fistula pouch is seen located in the anterior epidural space (B and C). This is better demonstrated on the axial angio-CT image $(C)$ where there is definite engorgement of the ventral epidural venous plexus. This is a feature that is not present among spinal dural arteriovenous fistulas. D-F: Spinal angiogram (A) and XperCT images (B and C) demonstrating a SDAVF fed by the right L-1 lumbar artery. Notice the location of the arteriovenous fistulous connection under the right $L-1$ pedicle ( $E$ and $F$ ). This is at the expected location of the nerve root sleeve, which is where these fistulas classically form.

dural space, and full exposure of this area from a posterolateral surgical approach can be cumbersome and associated with brisk epidural bleeding. Additionally, identification of the specific subtype is critical to prevent recurrence. In fact, in the presence of an SEDAVF, mere disconnection of the intradural draining vein without addressing the extradural fistula will often result in persistence of the extradural fistulous component with secondary recruitment of intradural veins at a level above or below the surgical site, with recurrence of the fistula. We suspect that some cases of recurrent fistula after surgical disconnection of a presumed Type I DAVF and some of the reported cases of multiple fistulous connections were indeed unrecognized SEDAVFs. ${ }^{20}$
The therapeutic goal in treating SEDAVFs is to obliterate the fistulous connection between the feeding artery and draining vein in the epidural space. In $75 \%$ of our cases, endovascular approaches alone were sufficient in treating these lesions. This is consistent with what has been reported in the literature, as currently endovascular treatment is the favored method of treatment, with about two-thirds of SEDAVF patients described in the literature being treated with endovascular approaches alone. ${ }^{8}$ High rates of complete and near-complete occlusion have been reported in the literature (about 90\%), similar to our findings. ${ }^{8,11}$ We perform follow-up conventional spinal angiography as well as spinal MR angiography in all of our patients treated with endovascular embolization to confirm

TABLE 2. Treatment and clinical outcomes

\begin{tabular}{lcccc}
\hline \multicolumn{1}{c}{ Variable } & Total & Endovascular & Surgical & Combined \\
\hline No. of patients & $24(100.0)$ & $18(75.0)$ & $4(16.7)$ & $2(8.3)$ \\
\hline Complete/near-complete occlusion & $22(91.7)$ & $17(94.4)$ & $3(75.0)$ & $2(100.0)$ \\
\hline Minor complication & $1(4.2)$ & $1(4.2)$ & $0(0.0)$ & $0(0.0)$ \\
\hline Major complication & $1(4.2)$ & $1(4.2)$ & $0(0.0)$ & $0(0.0)$ \\
\hline Improvement in disability & $15(62.5)$ & $12(66.7)$ & $1(25.0)$ & $2(100.0)$ \\
\hline Improvement in motor function & $15(62.5)$ & $12(66.7)$ & $1(25.0)$ & $2(100.0)$ \\
\hline Improvement in bowel Sx & $5(33.3)$ & $3(30.0)$ & $1(33.3)$ & $1(50.0)$ \\
\hline Improvement in sensory Sx & $12(54.5)$ & $9(56.3)$ & $1(25.0)$ & $2(100.0)$ \\
\hline Improvement in bladder Sx & $7(41.2)$ & $5(41.7)$ & $1(33.3)$ & $1(50.0)$ \\
\hline
\end{tabular}

Values represent numbers of patients (\%). 
TABLE 3. Comparison of pre- and posttreatment mRS and Aminoff scores

\begin{tabular}{ccc}
\hline Measure & Pretreatment & Posttreatment $^{*}$ \\
\hline mRS & & \\
\hline 0 & $0(0.0)$ & $2(8.3)$ \\
\hline 1 & $4(16.7)$ & $7(29.2)$ \\
\hline 2 & $5(20.8)$ & $5(20.8)$ \\
\hline 3 & $5(20.8)$ & $6(25.0)$ \\
\hline 4 & $10(41.7)$ & $4(16.7)$ \\
\hline 5 & $0(0.0)$ & $0(0.0)$ \\
\hline Aminoff & & $6(25.0)$ \\
\hline 0 & $3(12.5)$ & $3(12.5)$ \\
\hline 1 & $3(12.5)$ & $2(8.3)$ \\
\hline 2 & $2(8.3)$ & $4(16.7)$ \\
\hline 3 & $6(25.0)$ & $7(29.2)$ \\
\hline 4 & $3(12.5)$ & $2(8.3)$ \\
\hline 5 & $7(29.2)$ &
\end{tabular}

Data are presented as number of patients (\%).

* At most recent clinical follow-up visit.

persistent occlusion of the fistula. Follow-up MRI can be useful as well to monitor for changes in cord signal. Any progression in cord signal following embolization should prompt additional angiographic imaging to determine whether there is a recurrence of the fistula.

Reported clinical symptom improvement rates are $80 \%-90 \%$ with endovascular treatment alone, and about $5 \%$ of patients report worsening of symptoms. In our series, about two-thirds of the patients had improvement in disability following endovascular treatment, which is likely a reflection of the fact that our institution is a tertiary referral center and many patients experienced a long delay in diagnosis prior to treatment.

Endovascular treatment of SEDAVFs with intradural venous drainage is focused on occlusion of the venous pouch along with the proximal intradural draining vein. If the proximal pouch is not fully obliterated, recanalization can occur secondary to de novo recruitment of new arterial feeders. ${ }^{21}$ The primary complication of transarterial embolization is embolization of the radiculomedullary artery, which occurred in 1 case in our series. Selective angiography at a few levels above and below the actual connection to identify the anterior spinal artery is essential. It is also important to consider that the lack of angiographic visualization of vascular cord supply does not exclude the possibility of vascular supply from the same pedicle feeding the SEDAVF. Small posterior spinal arteries ascending with the corresponding nerve root are often present at many segments but may not necessarily be visualized. Large venous varices and pouches can complicate embolization due to the large amount of embolic material required to achieve complete obliteration. This is a particular problem in the treatment of SEDAVFs with pure extradural venous drainage, for which symptom onset often occurs once the venous pouch has become large enough to produce mass effect.

While transarterial embolization with liquid embolic agents is often the preferred treatment modality, transvenous embolization of SEDAVFs can also be performed. ${ }^{8,11}$ Transvenous embolization has been reported to be more effective than transarterial embolization in the setting of multiple arterial feeders. A unique approach to transvenous embolization was reported by Ramanathan et al., ${ }^{16}$ who performed direct percutaneous embolization of an epidural venous pouch with n-BCA (n-butyl cyanoacrylate) glue. In our series, 1 patient underwent percutaneous embolization of an epidural venous pouch.

Surgical treatment is generally reserved for lesions that are difficult or not safe to treat with endovascular management or for cases in which endovascular treatment has failed. With surgical treatment, long-term complete occlusion rates in the literature are in the range of $80 \%-90 \%$. In our series, of the 4 patients initially treated with surgery, 3 had complete obliteration of the fistula and 1 patient required further treatment with endovascular embolization with Onyx following a recurrence. Surgical treatment typically consists of direct ligation and interruption of the fistula at the site of the shunt and draining vein. ${ }^{5}$ One advantage of surgical treatment is the opportunity to perform both decompression and ligation thus reducing the SEDAVF associated mass effect. This treatment strategy is particularly useful in lesions without intradural venous drainage.

\section{Limitations}

There are a few limitations with this study. This is a retrospective review performed at a tertiary referral institution, which predisposes the study to selection bias. Based on our institution's experience, most of these patients had symptoms for a long duration of time due to either misdiagnosis or slow progression of symptoms, which could result in a higher rate of disability in our series. It is possible that patients who were diagnosed more quickly at their local institutions could have had better clinical outcomes. Another limitation is the fact that symptoms, disability rates, and motor function were assessed through a retrospective chart review. It is possible that the degree of disability could be overestimated or underestimated based on assessment of clinical notes. Lastly, the duration of clinical follow-up was variable among patients, ranging from 3 to 60 months. Further long-term follow-up is needed to confirm whether our treatments were indeed effective.

\section{Conclusions}

SEDAVFs often present with protean clinical manifestations secondary to congestive myelopathy. Due to their protean clinical manifestations, they are often misdiagnosed for other causes of myelopathy. While SEDAVFs share some clinical and imaging similarities with the more common Type I SDAVFs, there are also important differences, which have important therapeutic ramifications. With advances in endovascular techniques and better understanding of the pathological anatomy of SEDAVFs, endovascular embolization with liquid embolic agents has become the mainstay of therapy at our institution. Surgery is also effective at treating these lesions, particularly in situations of failed or unsafe endovascular treatment 
or for those rare patients who present with symptoms of mass effect from an enlarged epidural venous pouch. Motor symptoms show the highest rate of improvement following successful treatment, while sensory symptoms and bowel/bladder dysfunction improve only in one-half and one-quarter of patients, respectively.

\section{References}

1. Aminoff MJ, Logue V: The prognosis of patients with spinal vascular malformations. Brain 97:211-218, 1974

2. Brinjikji W, Nasr DM, Morris JM, Rabinstein AA, Lanzino $\mathrm{G}$ : Clinical outcomes of patients with delayed diagnosis of spinal dural arteriovenous fistulas. AJNR Am J Neuroradiol 37:380-386, 2016

3. Brinjikji W, Yin R, Nasr DM, Lanzino G: Spinal epidural arteriovenous fistulas. J Neurointerv Surg [epub ahead of print], 2016

4. Cabral AJ, Barros A, Aveiro C, Vasconcelos R: Spontaneous spinal epidural haematoma due to arteriovenous malformation in a child. BMJ Case Rep 2011:bcr0220113875, 2011

5. Chaudhary N, Pandey AS, Gemmete JJ: Endovascular treatment of adult spinal arteriovenous lesions. Neuroimaging Clin N Am 23:729-747, 2013

6. Clarke MJ, Patrick TA, White JB, Cloft HJ, Krauss WE, Lindell EP, et al: Spinal extradural arteriovenous malformations with parenchymal drainage: venous drainage variability and implications in clinical manifestations. Neurosurg Focus 26(1):E5, 2009

7. Elkordy A, Endo T, Sato K, Sonoda Y, Takahashi A, Tominaga T: Exclusively epidural spinal metameric arteriovenous shunts: case report and literature review. Spine J 15:e15-e22, 2015

8. Huang W, Gross BA, Du R: Spinal extradural arteriovenous fistulas: clinical article. J Neurosurg Spine 19:582-590, 2013

9. Khaldi A, Hacein-Bey L, Origitano TC: Spinal epidural arteriovenous fistula with late onset perimedullary venous hypertension after lumbar surgery: case report and discussion of the pathophysiology. Spine (Phila Pa 1976) 34:E775-E779, 2009

10. Kitagawa RS, Mawad ME, Whitehead WE, Curry DJ, Luersen TG, Jea A: Paraspinal arteriovenous malformations in children. J Neurosurg Pediatr 3:425-428, 2009

11. Lanzino G, D’Urso PI, Kallmes DF, Cloft HJ: Onyx embolization of extradural spinal arteriovenous malformations with intradural venous drainage. Neurosurgery 70:329-333, 2012

12. Marovic P, Thani N, Lu S, Bala A: Spinal subarachnoid hemorrhage secondary to rupture of an isolated radicular artery aneurysm. J Neurol Surg A Cent Eur Neurosurg 74:410414,2013

13. Miyamoto N, Naito I, Takatama S, Iwai T, Tomizawa S, Inoue HK: Spinal epidural arteriovenous fistulas with unusual manifestation of sudden onset of severe neurological deficits: case report. Neurol Med Chir (Tokyo) 53:896-901, 2013

14. Olivero WC, Hanigan WC, McCluney KW: Angiographic demonstration of a spinal epidural arteriovenous malformation. Case report. J Neurosurg 79:119-120, 1993

15. Paraskevopoulos D, Magras I, Polyzoidis K: Spontaneous spinal epidural hematoma secondary to extradural arteriovenous malformation in a child: a case-based update. Childs Nerv Syst 29:1985-1991, 2013

16. Ramanathan D, Levitt MR, Sekhar LN, Kim LJ, Hallam DK, Ghodke BV: Management of spinal epidural arteriovenous fistulas: interventional techniques and results. J Neurointerv Surg 6:144-149, 2014

17. Rispoli R, Mastrostefano R, Passalacqua G, Filauri P, Fontana M: Epidural hematoma caused by a spinal osseous epidural arteriovenous fistula in a 14-year-old patient. A case report. Neuroradiol J 22:452-457, 2009

18. Romeo F, Toscano S, Santangelo M, Fumai V, Maddalena G: Spontaneous cervical extradural hematoma in a cutaneomeningospinal angiomatosis (Cobb syndrome): case report. J Neurosurg Sci 53:59-61, 2009

19. Saeheng S, Thongsuksai P: Spinal epidural hematoma caused by extradural arteriovenous malformation: a case report and review of the literature. J Med Assoc Thai 82:520-527, 1999

20. Saladino A, Atkinson JL, Rabinstein AA, Piepgras DG, Marsh WR, Krauss WE, et al: Surgical treatment of spinal dural arteriovenous fistulae: a consecutive series of 154 patients. Neurosurgery 67:1350-1358, 2010

21. Silva N Jr, Januel AC, Tall P, Cognard C: Spinal epidural arteriovenous fistulas associated with progressive myelopathy. Report of four cases. J Neurosurg Spine 6:552-558, 2007

22. Solero CL, Fornari M, Savoiardo M: Spontaneous spinal epidural haematoma arising from ruptured vascular malformation. Case report. Acta Neurochir (Wien) 53:169-174, 1980

23. Takai K, Taniguchi M: Comparative analysis of spinal extradural arteriovenous fistulas with or without intradural venous drainage: a systematic literature review. Neurosurg Focus 32(5):E8, 2012

\section{Disclosures}

Dr. Lanzino reports a consultant relationship with Covidien/ Medtronic.

\section{Author Contributions}

Conception and design: Nasr, Brinjikji, Lanzino. Acquisition of data: Nasr, Brinjikji. Analysis and interpretation of data: Nasr, Lanzino. Drafting the article: Nasr, Brinjikji. Critically revising the article: all authors. Reviewed submitted version of manuscript: all authors. Approved the final version of the manuscript on behalf of all authors: Nasr. Statistical analysis: Nasr, Brinjikji. Administrative/technical/material support: Nasr. Study supervision: Lanzino.

\section{Correspondence}

Deena M. Nasr, Department of Neurology, Mayo Clinic, 200 1st St. SW, Rochester, MN 55905. email: nasr.deena@mayo.edu. 